\title{
Early prediction of the long term evolution of multiple sclerosis: the Bayesian Risk Estimate for Multiple Sclerosis (BREMS) score
}

\author{
Roberto Bergamaschi, Silvana Quaglini, Maria Trojano, Maria Pia Amato, Eleonora Tavazzi, \\ Damiano Paolicelli, Valentina Zipoli, Alfredo Romani, Aurora Fuiani, Emilio Portaccio, Carlo \\ Berzuini, Cristina Montomoli, Stefano Bastianello, Vittorio Cosi
}

\begin{abstract}
Aim: To propose a simple tool for early prediction of unfavourable long term evolution of multiple sclerosis (MS). Methods: A Bayesian model allowed us to calculate, within the first year of disease and for each patient, the Bayesian Risk Estimate for MS (BREMS) score that represents the risk of reaching secondary progression (SP).

Results: The median BREMS scores were higher in 158 patients who reached SP within 10 years compared with 1087 progression free patients $(0.69$ vs $0.30 ; p<0.0001)$. The BREMS value was related to SP risk in the whole cohort $(p<0.0001)$ and in the subgroup of 535 patients who had never been treated with immune therapies, thus reasonably representing the natural history of the disease $(p<0.000001)$. Conclusions: The BREMS score may be useful both to identify patients who are candidates for early or for more aggressive therapies and to improve the design and analysis of clinical therapeutic trials and of observational studies.
\end{abstract}

$\mathrm{T}$ he widespread and early use of disease modifying drugs in multiple sclerosis (MS), such as $\beta$-interferons and glatiramer acetate, is becoming more and more popular, ${ }^{1}$ even though patients with a favourable disease evolution could avoid these lifelong medications. ${ }^{2}$ It is therefore crucial to have reliable clinical predictors of the evolution of the disease to distinguish between patients requiring early/aggressive therapies from those who do not, and to improve the design and analysis of clinical therapeutic trials and of observational studies.

Several studies have identified clinical factors related to poor MS outcome. ${ }^{3-9}$ However, these studies analysed the relationships between manifestations at onset and time of occurrence of specified end points, without considering information collected up to the end points. To make more accurate use of follow-up information and to better assess the prognostic value of the early events, we performed a preliminary study by building a Bayesian model of the natural history of the disease. ${ }^{10}$ The model allowed us to calculate an individual risk score for each patient.

In the present study we tested the trustworthiness of the Bayesian risk score on the basis of a new and larger sample of patients.

\section{METHODS}

In a previous study, we exploited a Bayesian approach with a Markov chain Monte Carlo simulation to model the natural history of 186 MS patients with an initial relapsing-remitting (RR) course in order to determine the risk of reaching secondary progression (SP). ${ }^{10}$ This risk was significantly related to specific clinical factors collected during the first year of the disease. Each factor was associated with a specific statistical "weight", the Bayesian local relative risk (LRR), which we used to calculate the Bayesian Risk Estimate for MS (BREMS) score. The BREMS score can be simply calculated, for any given patient, from the sum of the appropriate log LRRs (table 1).

For example, if we consider a 50-year-old woman, who had a "motor-sensory" onset without complete recovery, followed by one "sphincter plus motor relapse" which led to a permanent level 4.0 on the Expanded Disability Status Scale (EDSS) ${ }^{11}$ during the first year of disease, her BREMS score would be: 0.25 (age: $0.05 \times 5$ decades) -1.07 (female sex) +0.81 (motorsensory involvement at onset) +0.64 (number of functional systems involved at onset: 2$)+0.52$ (sequel after onset) +0.71 ( sphincter plus motor relapse) $+0.44($ EDSS $=4.0$ outside relapse) $=2.30$.

We retrospectively analysed the prospectively collected data of a hospital cohort of MS patients selected on the basis of the following criteria: diagnosis of definite MS according to Poser and colleagues ${ }^{12}$; initial RR course; disease duration $\geqslant 10$ years; and interval from clinical onset to the first neurological examination $\leqslant 1$ year.

We analysed the clinical variables collected at the initial observation and within 12 months of disease onset, at each scheduled or unscheduled visit. We recorded the time at which the patient reached the confirmed SP. SP was defined as the earliest date of observation of a progressive worsening, severe enough to lead to an increase of at least 1 point on the EDSS, and confirmed at least 1 year after progression. ${ }^{13}$

\section{RESULTS}

We enrolled 1245 MS patients (863 females, 382 males) selected from a larger sample of 3373: 162 patients, who were different from those who contributed to the Bayesian model in the preliminary study, ${ }^{10}$ came from the MS Centre in Pavia (Northern Italy), 295 patients from the MS Centre in Florence (Central Italy) and 788 from the MS Centre in Bari (Southern Italy).

Median age at onset was 24.8 years (interquartile range (IQR) 20-32)) and mean follow-up was 17.1 years (SD (2.1), range 10-48); 21261 person years were analysed.

A total of 340 patients $(27 \%)$ reached the main end point ( shift to SP) by the end of the entire observation period, in a median time of 10.5 years (range 2-44).

In all, 710 patients were treated with at least one immune therapy (disease modifying drugs, azathioprine, cyclophosphamide,

Abbreviations: BREMS, Bayesian Risk Estimate for Multiple Sclerosis; EDSS, Expanded Disability Status Scale; LRR, local relative risk; MS, multiple sclerosis; RR, relapsing-remitting; SP, secondary progression 
Table 1 Estimates of the Bayesian risk associated with early clinical predictors observed within 1 year of disease onset

\begin{tabular}{|c|c|c|c|}
\hline & $\begin{array}{l}\text { Mean } \\
\text { LRR }\end{array}$ & $\begin{array}{l}\text { Mean log } \\
\text { LRR }\end{array}$ & $95 \% \mathrm{Cl}$ \\
\hline Age at onset (in decades) & 1.05 & 0.05 & 1.02 to 1.09 \\
\hline Female sex & 0.39 & -1.07 & 0.17 to 0.78 \\
\hline Sphincter onset & 2.98 & 0.93 & 1.10 to 6.10 \\
\hline Pure motor onset & 2.11 & 0.62 & 0.90 to 4.20 \\
\hline Motor-sensory onset & 2.40 & 0.81 & 1.15 to 4.41 \\
\hline Sequel after onset & 1.76 & 0.52 & 1.04 to 2.88 \\
\hline $\begin{array}{l}\text { Functional systems involved at } \\
\text { onset }\end{array}$ & 1.39 & 0.32 & 1.16 to 1.64 \\
\hline Sphincter plus motor relapses & 2.10 & 0.71 & 1.56 to 2.89 \\
\hline EDSS $\geqslant 4$ outside relapse & 2.28 & 0.44 & 0.40 to 6.50 \\
\hline
\end{tabular}

EDSS, Expanded Disability Status Scale; LRR, local relative risk; $95 \%$ Cl, $95 \%$ Bayesian credible interval for the local relative risk.

ciclosporin, mitoxantrone). The remaining 535 patients were never treated, except with steroids during relapses. Among the patients never treated, 179 (33\%) became SP by the end of the entire observation period, in a median time of 10.1 years (range 2-34).

We calculated the BREMS score, based on the clinical events of the first year of disease, for every patient.

We divided patients into two groups, on the basis of the clinical course observed during the first 10 years from disease onset: 1087 patients were RR and 158 SP. The median BREMS score was significantly higher in the subgroup of patients who reached SP within 10 years than in the progression free patients: 0.69 (IQR -0.08 to 1.31 ) vs 0.30 (IQR -0.58 to $0.94)$, Mann-Whitney test $\mathrm{z}=5.6, \mathrm{p}<0.0001$.

We then focused our analyses on the 535 patients who were never treated with immune therapies, and who thus represented the natural history of the disease more closely. Their clinical course within 10 years was RR in 448 and SP in 87. Again, the median BREMS score was significantly higher in the subgroup of patients who reached SP: 0.82 (IQR 0.11 to 1.51 ) vs 0.28 (IQR -0.58 to 0.93 ), Mann-Whitney test $\mathrm{z}=5.2$, $\mathrm{p}<0.0001$. In contrast, we did not find any significant difference in BREMS scores when we analysed 710 patients (639 RR and 71 SP) treated with at least one immune therapy.

SP-free survival analysis also demonstrated that BREMS was significantly related to SP in the whole cohort $(\mathrm{p}<0.0001)$ and in the "never treated cohort" $(\mathrm{p}<0.0001)$ but not in the "treated cohort".

We subsequently divided patients on the basis of quartiles, and compared patients with high scores (fourth quartile, BREMS value $\geqslant 0.97$ ) to patients with low scores (first quartile, BREMS value $\leqslant-0.55)$. Among the patients with higher BREMS scores, $29 \%$ became SP within 10 years of disease onset while only $4 \%$ of those with a lower BREMS score became SP. The relative risk was 6.5 (95\% CI 2.8 to 14.8). Kaplan-Meier curves confirmed that a higher BREMS value was significantly related to a higher risk of reaching SP in the whole cohort $(\mathrm{p}<0.0001)$ and in the subgroup of patients who were never treated $(\mathrm{p}<0.000001)$ (fig 1$)$, while no significant difference was found for the treated subgroup.

Finally, we regarded BREMS as a "diagnostic" test with which to predict (i) the risk of reaching SP and (ii) the probability of being progression free at 10 years from clinical onset. We analysed patients who started with a BREMS score $\geqslant 95$ th percentile (value 2.0) and those who started with a BREMS score $\leqslant 5$ th percentile (value -0.63 ). Of the 21 "very high risk" patients, 18 became SP; specificity was 0.99 , sensitivity 0.17 , positive predictive value 0.86 and negative predictive value 0.83 . All 33 of the "very low risk" patients

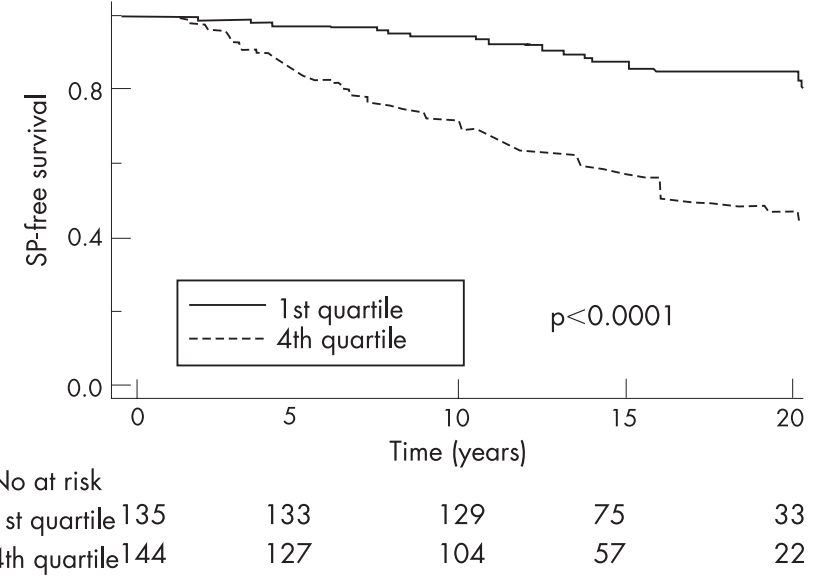

Figure 1 Kaplan-Meier curves for estimates of the time needed to reach the secondary progressive (SP) phase, starting from the 1 year duration of disease mark, among patients with multiple sclerosis who were never treated with immune therapies, with Bayesian Risk Estimate for Multiple Sclerosis values $\leqslant 1$ st quartile or $\geqslant 4$ th quartile.

remained progression free; specificity was 1.00 , sensitivity 0.08 , positive predictive value 1.00 and negative predictive value 0.18 .

\section{DISCUSSION}

In the present study, we tested a method for the early prediction of long term poor evolution of MS, namely the development of SP. This latter event represents a critical step towards an irreversible disability for which currently available immune therapies are no longer useful.

We used the prognostic factors we had previously selected with a Bayesian model of the natural history of $\mathrm{MS}^{10}$ to calculate, in the first year of disease, a risk score (the BREMS) for each individual patient. We analysed patient data from three independent Italian MS Centres to determine whether the BREMS score accurately predicted the actual evolution of the disease.

Regarding the statistical approach on which we based our analysis, Bayesian methodology has, in recent years, been promoted as an alternative to the frequentist method when dealing with medical problems, ${ }^{14}$ such as MS prognosis. ${ }^{15}$ The Bayesian approach not only confirmed the findings of other studies (the "protective" effect of female sex, ${ }^{4-68}$ the unfavourable role of late age at onset and of polysymptomatic onset ${ }^{45}$ ) but also highlighted the importance of the qualitative aspects of clinical events. Specifically, type of onset, motor and sphincter relapses, and an early increase in disability were important short term predictors of long term outcome. This suggests that we should look at not only the number of events but should also consider the relative importance of their different characteristics.

Our analyses showed that the BREMS score can predict disease evolution. In addition to analysing the whole cohort of 1245 MS patients, we separately tested 535 patients who had never been treated with immune therapies. We confirmed the predictive capability of BREMS in this cohort, which seemed to better represent the natural history of the disease. Analysis of the 710 patients treated with at least one immune therapy generated further speculation. Given that the present study was not designed to evaluate the effect of treatment (and thus we provided no specific analyses concerning type, duration, concomitance or sequence of therapies), we found no difference in reaching SP despite initial differences in BREMS. Thus treatment seems to bring disease evolution in high risk patients 
in line with that of low risk patients, which in turn suggests that immune therapy is favourable in the long term.

As a diagnostic test, BREMS had poor sensitivity; it needs improvement through incorporation of additional clinical aspects of the disease (such as cognitive impairment and fatigue), as well as genetic, neuroimmunological, neuroradiological and neurophysiological findings. Nevertheless, the present test is extremely specific. In particular, patients with an initial BREMS score $>2.0$ have a high probability of reaching SP within 10 years while patients with a BREMS value $<-0.63$ are very likely to remain progression free. Although immune treatment in all patients with a diagnosis of RR MS is considered appropriate, we should recognise that patients destined to have a favourable course would benefit from not receiving treatments. With BREMS available for every patient, early treatment or very efficient but relatively unsafe therapies (such as natalizumab) could be reserved for high risk patients, a wait and see approach could be adopted for low risk cases and the use of immunomodulators could be considered in the remaining patients.

In conclusion, we propose the BREMS score as a simple tool for the early prediction of the long term evolution of MS. BREMS may be useful to: (i) identify high risk patients who require early or more aggressive therapies; (ii) identify low risk patients who could avoid lifelong, expensive and potentially troublesome treatments; (iii) favour a more homogeneous selection of patients for clinical therapeutic trials, where inclusion criteria such as the simple relapse count are still applied; and (iv) evaluate the effect of therapies in the field of observational studies, facilitating an a posteriori subdivision of non-randomised patients on the basis of their different "propensity" to reach a poor end point.

\section{Authors' affiliations \\ Roberto Bergamaschi, Eleonora Tavazzi, Alfredo Romani, Stefano \\ Bastianello, Vittorio Cosi, Multiple Sclerosis Centre, Neurological Institute C Mondino of Pavia, Pavia, Italy \\ Silvana Quaglini, Carlo Berzuini, Department of Informatics and System Sciences, University of Pavia, Pavia, Italy \\ Maria Trojano, Damiano Paolicelli, Aurora Fuiani, Department of Neurological and Psychiatric Sciences, University of Bari, Bari, Italy Maria Pia Amato, Valentina Zipoli, Emilio Portaccio, Department of Neurological and Psychiatric Sciences, University of Florence, Florence, Italy}

Cristina Montomoli, Department of Health Sciences, Section of Medical Statistics and Epidemiology, University of Pavia, Pavia, Italy

Competing interests: None.

Correspondence to: Dr Roberto Bergamaschi, Multiple Sclerosis Centre, Department of Clinical Neurology, Neurological Institute "C Mondino", Via Mondino 2, 27100 Pavia, Italy; roberto.bergamaschi@mondino.it

Received 17 September 2006

Revised 28 November 2006

Accepted 4 January 2007

Published Online First 12 January 2007

\section{REFERENCES}

1 Flachenecker $\mathbf{P}$. Disease-modifying drugs for the early treatment of multiple sclerosis. Expert Rev Neurother 2004;4:455-63.

2 Pittock SJ, Weinshenker BG, Noseworthy JH, et al. Not every patient with multiple sclerosis should be treated at time of diagnosis. Arch Neurol 2006;63:611-14

3 Weinshenker BG, Bass B, Rice GPA, et al. The natural history of multiple sclerosis: a geographically based study. 2. Predictive value of the early clinical course. Brain 1989:112:133-46.

4 Phadke JC. Clinical aspect of multiple sclerosis in North East Scotland with particular reference to its course and prognosis. Brain 1990;113:1597-628.

5 Runmarker B, Andersen O. Prognostic factors in a multiple sclerosis incidence cohort with twenty five years of follow-up. Brain 1993;116:117-34.

6 Trojano M, Avolio C, Manzari C, et al. Multivariate analysis of predictive factors of multiple sclerosis course with a validated method to assess clinical events. J Neurol Neurosurg Psychiatry 1995;58:300-6.

7 Amato MP, Ponziani G, Bartolozzi ML, et al. A prospective study on the natural history of multiple sclerosis: clues to the conduct and interpretation of clinical trials. J Neurol Sci 1999;168:96-106.

8 Confavreux C, Vukusic S, Adeleine P. Early clinical predictors and progression of irreversible disability in multiple sclerosis: an amnesic process. Brain 2003; 126:770-82.

9 Ebers GC. Prognostic factors for multiple sclerosis: the importance of natural history studies. J Neurol 2005;252(Suppl 3):iii15-20.

10 Bergamaschi R, Berzuini C, Romani A, et al. Predicting secondary progression in relapsing-remitting multiple sclerosis: a Bayesian analysis. J Neurol Sci 2001;189:13-21.

11 Kurtzke JF. Rating neurologic impairment in multiple sclerosis: an expanded disability status scale (EDSS). Neurology 1983;33:1444-52.

12 Poser CM, Paty DW, Sheinberg L, et al. New diagnostic criteria for multiple sclerosis: guidelines for research protocols. Ann Neurol 1983;13:227-31.

13 Kremenchutzky M, Cottrell D, Rice G, et al. The natural history of multiple sclerosis: a geographically based study. 7. Progressive-relapsing and relapsingprogressive multiple sclerosis: a re-evaluation, Brain 1999;122:1941-50.

14 Berzuini C, Larizza C. A unified approach for modelling longitudinal and failure time data, with application in medical monitoring. IEEE Trans Patt An Mac Intell 1996;2:109-23.

15 Weinshenker BG. Bayesian analysis: what does it add to studies of the natural history of MS? J Neurol Sci 2001;189:1-2. 